\title{
Strategische Personalplanung und HR Analytics
}

\section{Navigationshilfe für das Management am Beispiel der AOK Hessen}

\author{
Kai Berendes $\cdot$ Johannes Kumpf • Marc Delarue
}

Eingegangen: 31. Juli 2016 / Angenommen: 26. September 2016 / Online publiziert: 13. Oktober 2016 (C) Der/die Autor(en) 2016. Dieser Artikel ist eine Open-Access-Publikation.

Zusammenfassung Die Veränderungsgeschwindigkeit von Geschäftsdynamik und Personaldynamik läuft immer stärker auseinander. Umfassende Human Resource (HR) Analytics konzentriert sich deshalb nicht nur auf das „Hier und Jetzt“", sondern nimmt mit einer integrierten strategischen Personalplanung eine zukunftsgerichtete Perspektive ein. Sie bildet die wichtige Brücke zwischen Geschäftsstrategie und Personalmanagement. Dargestellt am Beispiel der AOK Hessen können Personalmanager mit Hilfe von Szenariosimulation die richtigen Initiativen heute einleiten und mit ihrer Wirkung das Mitarbeiterportfolio entlang der Anforderungen aus dem Geschäft für die Zukunft sicher gestalten. Dabei wird die Personalfunktion ganz von alleine zum Business Partner.

Schlüsselwörter Human Resource Management - Strategische Personalplanung · People Analytics, Szenariosimulation · Digitalisierung · Risikomanagement

\section{Strategic Workforce Planning and HR Analytics}

A Pathfinder for the Management at AOK Hessen

Abstract Business and Human Resources (HR) are running more and more at different speed. Therefore, progressive HR analytics must not focus on an "inside

\footnotetext{
K. Berendes $(\square)$

Dynaplan AG, St. Gallen, Schweiz

E-Mail: kai.berendes@dynaplan.com

J. Kumpf

Weysen GmbH (a CubeServ Company), Raunheim, Deutschland

E-Mail: Johannes.Kumpf@weysen.com

M. Delarue

AOK Hessen, Offenbach, Deutschland

E-Mail: Marc.Delarue@he.aok.de
} 
and now view" only. The integration with strategic workforce planning will complete the view by adding the long-term perspective of employee development into the future. Strategic workforce planning builds the bridge between business strategy and HR. In the case of AOK Hessen decision makers in HR will - based on scenario simulation - derive today's initiatives for shaping the future workforce ready for implementing business strategy. By following this approach HR becomes a real business partner.

Keywords Human Resource Management - Strategic Workforce Planning · People Analytics, Scenario Simulation · Digital Transformation · Risk Management

\section{Strategische Personalplanung im Kontext von HR Analytics}

Zahlen, Daten und Fakten sind unmittelbar mit gutem Personalmanagement verbunden. Schon lange nicht mehr werden mit HR-Funktionen nur scheinbar weiche Themen wie Personalentwicklung verbunden. HR Analytics oder auch People Analytics wird in diesem Zusammenhang immer wieder als Schlagwort aufgegriffen. Je nach Definition lassen sich darunter eine Vielzahl von Methoden und Werkzeugen fassen, welche den Personalkörper in Unternehmen unter unterschiedlichen Perspektiven betrachten. Vergleichbar mit der Ausleuchtung eines verwinkelten Raumes sind mehrere Leuchtmittel respektive Werkzeuge notwendig, um ein umfassendes Bild zu erhalten. Das gilt insbesondere mit Blick auf die zeitliche Perspektive. Das Reporting beschreibt zunächst die aktuelle Situation sowie die Entwicklung der Vergangenheit. Auf dieser Basis können mit Hilfe statistischer Methoden kurzfristige Vorhersagen erstellt werden. Nicht immer ist jedoch die Vergangenheit ein guter Indikator für die Zukunft. Gerade in einem dynamischen Umfeld mit veränderlichen Rahmenbedingungen sind zusätzliche Ansätze erforderlich, diese Unbestimmtheit aufzugreifen. Eine strategische Personalplanung kann die Unbestimmtheit nicht auflösen. Sie erlaubt aber eine aktive Auseinandersetzung mit möglichen zukünftigen Entwicklungen und gibt mit der engen Orientierung an die Unternehmensstrategie der Personalfunktion die notwendige Vorbereitungszeit für den Anstoß der richtigen Initiativen. Schließlich sind gerade Personalentscheidungen in ihrer Wirkung hoch

Abb. 1 Strategische Personalplanung im Kontext von HR Analytics
Benchmarking

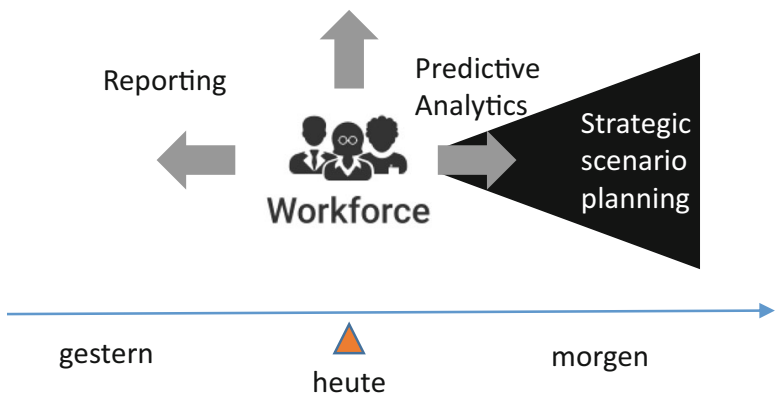


zeitverzögert. Themen der Aus- und Weiterbildung sind dabei nur ein bekanntes Beispiel. Es vergehen mehrere Jahre bevor die Wirkung sichtbar wird und neue Mitarbeiter in der gewünschten Qualifikation zur Verfügung stehen. Abgerundet wird das Bild (vgl. Abb. 1) durch die Außenperspektive. Der Blick nach links und rechts zum Beispiel im Rahmen vom Benchmarking erlaubt eine Einordnung der eigenen Situation.

\section{Ansatz der Strategischen Personalplanung}

Zielsetzung der strategischen Personalplanung ist die Sicherstellung der notwendigen Humanressourcen für die Umsetzung der Unternehmensstrategie. Sie setzt damit zunächst am Personalbedarf an. Dabei stehen die zukünftig benötigten Funktionen im Vordergrund und im Vergleich zu einer operativen Planung nicht der einzelne Mitarbeiter. Abgeleitet aus der Strategie werden die zentralen Geschäftstreiber (z. B. Volumentreiber der angebotenen Leistungen und auch die Produktivität im Rahmen der Leistungserstellung) in ihrer Wirkung auf den Personalbedarf über die Zeit beschrieben. Dem gegenüber steht die Entwicklung des Personalbestands. Seine Eigendynamik ergibt sich im Wesentlichen aus bekannten und unbekannten Abgängen sowie geplanten Zugängen. Da neben einer reinen Kapazitätsbetrachtung auch gerade die Mitarbeiterstruktur hinsichtlich der Aufteilung auf die unterschiedlichen Funktionen und Qualifikationsstufen relevant ist, kommt auch der Bewegungsdynamik innerhalb des Mitarbeiterbestands eine hohe Bedeutung zu. Aus Bedarf und Bestand ergibt sich die Personallücke (s. Abb. 2). Dabei lassen sich grundsätzlich zwei Richtungen unterscheiden: Auf der einen Seite geht es um den Ersatzbedarf, bedingt durch die Abgangsdynamik wie Alterung der Mitarbeiter und Fluktuation. Daneben entsteht ein Zusatzbedarf, welcher sich aus den Veränderungen z. B. der Volumentreiber auf der Bedarfsseite in den bestehenden Funktionen ergibt oder sich aus neuen Funktionen aufgrund der Anpassungen des Geschäftsmodells oder auch veränderten Prozessen und Technologien ableitet. Insbesondere diese beiden Aspekte unterstreichen, warum die strategische Personalplanung über eine Analyse des Personalbestands auf der Basis von Vergangenheitsdaten hinausgeht. Neue Funktionen wie beispielsweise ein „Digital Manager“ aufgrund von einem neu eingeführten digitalen Vertriebskanal blieben unberücksichtigt. Der Abgleich zwischen Bedarf und Bestand bildet die Ausgangsbasis für die Ableitung von notwendigen Maßnahmen. Dabei stehen wiederum auf beiden Seiten Stellhebel zur Verfügung. Entlang der HR-Wertekette stehen dabei verkürzt beschrieben für den Ausgleich der Personallücke die drei Felder Rekrutierung, Entwicklung und Freisetzung zur Verfügung. Auf der anderen Seite befinden sich auch Ansatzpunkte im Geschäftsfeld wie beispielsweise Entscheidungen über die Fertigungstiefe und damit verbundenen Einsatz von eigenen Mitarbeitern sowie auch die Prozessgestaltung und damit einhergehende Produktivität. Nach Peter Drucker, einem großen Managementvordenker, geht es im Management nicht um die Zukunft, sondern um die zukünftige Wirkung der heutigen Entscheidungen (Drucker 1993). Die zeitliche Wirkung der Maßnahmen ist demnach von erheblicher Bedeutung und bleibt nicht selten in vielen Ansätzen und Werkzeugen völlig ausgeblendet. Aber gerade dieser Aspekt ist 
Abb. 2 Balance zwischen Geschäfts- und Personaldynamik als Kernaufgabe der strategischen Personalplanung

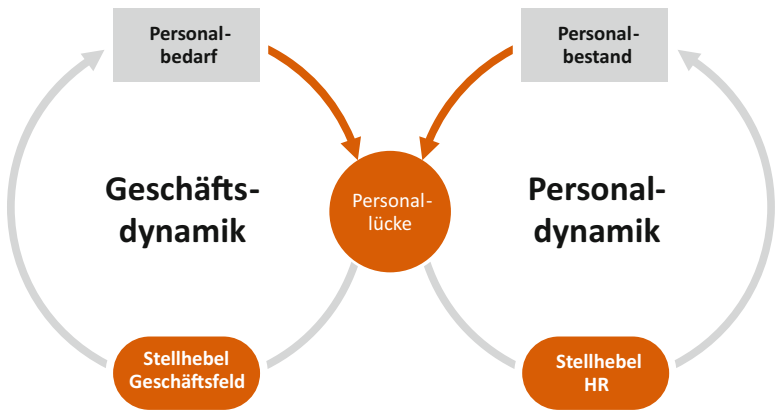

für die Bewertung von Optionen (mögliche Bündelung unterschiedlicher Stellhebel) neben einer monetären Sicht kritisch. Aus der Kognitionspsychologie weiß man schon lange um die Schwierigkeiten im Umgang mit komplexen Systemen (Dörner 1989). Ein Stolperstein ist die Fehleinschätzung der zeitlichen Dynamik und Rückkopplungsbeziehungen. In der Praxis zeigt sich das beispielsweise in fehlenden Ausgebildeten, da die Ausbildung aufgrund von Einsparungsmaßnahmen in vorangegangen Jahren ausgesetzt wurde, oder Überraschungen bei Expats, die nach ihrer Auslandstätigkeit völlig unerwartet wieder an die Tür klopfen und sich zurückmelden. Ein weiteres Beispiel sind ungeplante Anstellungen von ehemaligen Mitarbeitern z. B. in Form von Beratungsverträgen, da eine Nachfolgeregelung gefehlt hat. Über die Stellhebel schließen sich die beiden Kreisläufe in der Abb. 2 mit jeweils unterschiedlicher Geschwindigkeit. Im Gegensatz zur Geschäftsdynamik ist die Personaldynamik deutlich träger. Mehrere Jahre werden benötigt, um kurzfristige aus dem Fachbereich geforderte neue Qualifikationen aufzubauen. Entscheidend ist demnach für eine erfolgreiche Strategieumsetzung, diese unterschiedlichen Geschwindigkeiten auszubalancieren. Gesucht sind demnach Planungsmodelle, welche diese agile Sichtweise unterstützen.

\section{Umsetzung am Beispiel der AOK Hessen}

\subsection{Dynamisches Planungsmodell}

Dreh- und Angelpunkt für die Planung der AOK Hessen, eine Krankenkasse mit über 1,5 Mio. Versicherten und rund 90.000 Firmenkunden, ist ein dynamisches Planungsmodell, welches die Wirkungen der Kreisläufe über die Zeit nicht unterbricht und Bestands- und Bedarfsdynamik mit einer ganzheitlichen Sichtweise erfasst (vgl. Wegerich 2015). Hiermit lassen sich die Ziele, welche die AOK mit der strategischen Personalplanung verfolgt, erreichen. Die strategische Personalplanung

- unterstützt demnach eine mittel- und langfristige, quantitative und qualitative Personalprognose und schafft somit ein hohes Maß an Planungssicherheit,

- berücksichtigt die prognostizierte Marktentwicklung und bezieht, soweit absehbar, veränderte Rahmenbedingungen, beispielsweise durch den Gesetzgeber be- 
einflusst oder auch durch technologische Entwicklung wie u. a. Digitalisierung, in Form von Szenarien ein,

- untermauert einerseits notwendige Veränderungsentscheidungen angesichts eines bestimmten, vorgegebenen Haushaltsvolumens und zeigt andererseits die durch die Veränderung einzelner Parameter verursachten Personalkostenwirkungen auf,

- ermöglicht das ressourcenschonende Simulieren unterschiedlicher Szenarien unter Einbindung regionaler Besonderheiten.

Wichtiger Ausgangspunkt für die Simulation bilden die Daten und deren Qualität. Die einzelnen Planstellen werden dabei im Fall der AOK Hessen zu 60 Clustern zusammengefasst (siehe auch Abschn. 3.3). Neben der Initialisierung des Mitarbeiterbestands zum Startpunkt der Simulation helfen historische Daten bei der Bildung von Annahmen sowie möglichen Entscheidungen. Unter Annahmen werden aus systemtheoretischer Sicht Modellgrößen verstanden, welche außerhalb des eigenen Einflussbereichs liegen (externe Variablen) und nicht bzw. nicht direkt durch das Unternehmen beeinflusst werden können. Beispiele sind hierfür neben dem gesetzlichen Renteneintrittsalter auch Größen aus dem Marktumfeld wie bei der AOK Hessen z. B. die Versichertenentwicklung hinsichtlich Menge und Struktur. Direkt beeinflussbare Größen, in dem Modell als Entscheidungsvariablen bezeichnet, sind beispielsweise die Anzahl der jährlichen Auszubildenden oder auch externe Einstellungen. Idee hinter der Szenariosimulation ist es nun, verschiedene Werteausprägungen zu einem schlüssigen Zukunftsbild zu bündeln. Die externen Größen bestimmen dabei die Unsicherheit in den Entwicklungen und führen letztlich zu einem Ergebniskorridor (bekannt auch unter dem Stichwort Bandbreitenplanung). Modellstruktur, Daten, Annahmen sowie zukünftige Entscheidungen bestimmen zusammen das Ergebnis einer Szenariosimulation (siehe Abb. 3).

Abb. 3 Übersicht der Komponenten der Szenariosimulation

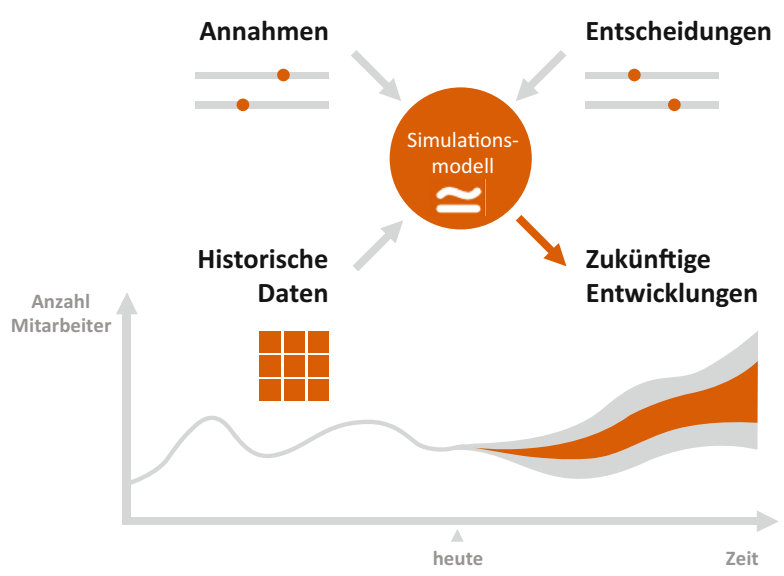




\subsection{Besonderheiten im Planungsmodell}

Die Komplexität eines Planungsmodells hängt zum einem vom Detaillierungsgrad der Informationen ab, welcher sich in der Anzahl der einbezogenen Faktoren widerspiegelt. Neben dieser sogenannten Varietät eines Systems treiben jedoch vor allem die Konnektivität und die Funktionalität eines Systems dessen Komplexität (Milling 2002). Die Konnektivität beschreibt wie stark das System durch Wechselbeziehungen zwischen den Faktoren geprägt ist und beeinflusst die Dynamik des Systems. Gerade diese Eigenschaft darf bei der Modellierung nicht unberücksichtigt bleiben. So werden beispielsweise Entwicklungspfade zwischen verschiedenen Qualifikationsleveln im Rahmen der Modellerarbeitung gemeinsam mit den Fachbereichen der AOK Hessen herausgearbeitet und im Modell abgebildet. Die Vielzahl der Treiber und deren Zusammenhänge stellen eine große Herausforderung bei der Modellerstellung dar. Ohne grafische Visualisierung gehen schnell Beziehungen verloren bzw. bleiben am Ende unberücksichtigt. Wichtig ist es daher neben der Datensicht (Tabellen) auch eine Sicht auf die Zusammenhänge zu erhalten (Treiberbäume und Wirkungsdiagramme). In der Abb. 4 wird die interne Weiterentwicklung von Mitarbeitern im Vergleich zu den anderen Zu- und Abgangsgrößen über ein kreisförmiges Flusssymbol dargestellt. Im Falle z. B. von einer Unterdeckung, wendet das Simulationsmodell die Entwicklungslogik an und füllt die Lücke auf. Unter Umständen führt diese Nachführung wiederum zu einer neuen Lücke in der Quellfunktion. Die Unterdeckung verschiebt sich demnach in andere Jobcluster und zeigt somit ein differenziertes Bild im Vergleich zu einer Lückenbetrachtung ohne Berücksichtigung von internen Entwicklungen wie beispielsweise in linearen Modellen abgebildet in Tabellenkalkulationsprogrammen. Für die Parametrisierung und die Analyse steht den Anwendern der AOK Hessen ein Simulationscockpit zur Verfügung (vgl. Abb. 5). Hier können schnell neue Parameterkombinationen eingegeben, über die Zeit exploriert und untereinander verglichen werden.

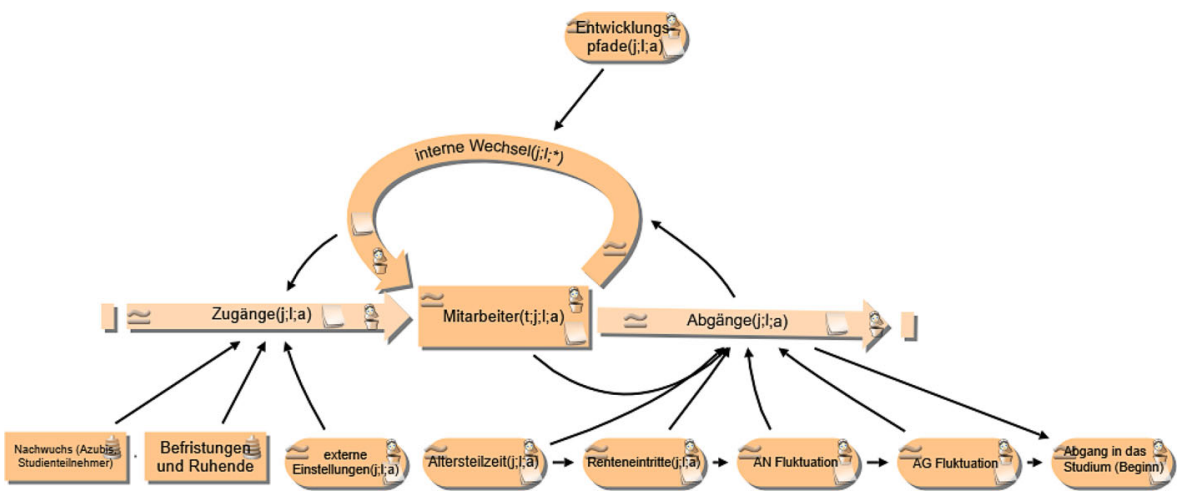

Abb. 4 Struktursicht auf den Mitarbeiterfluss am Beispiel Dynaplan 


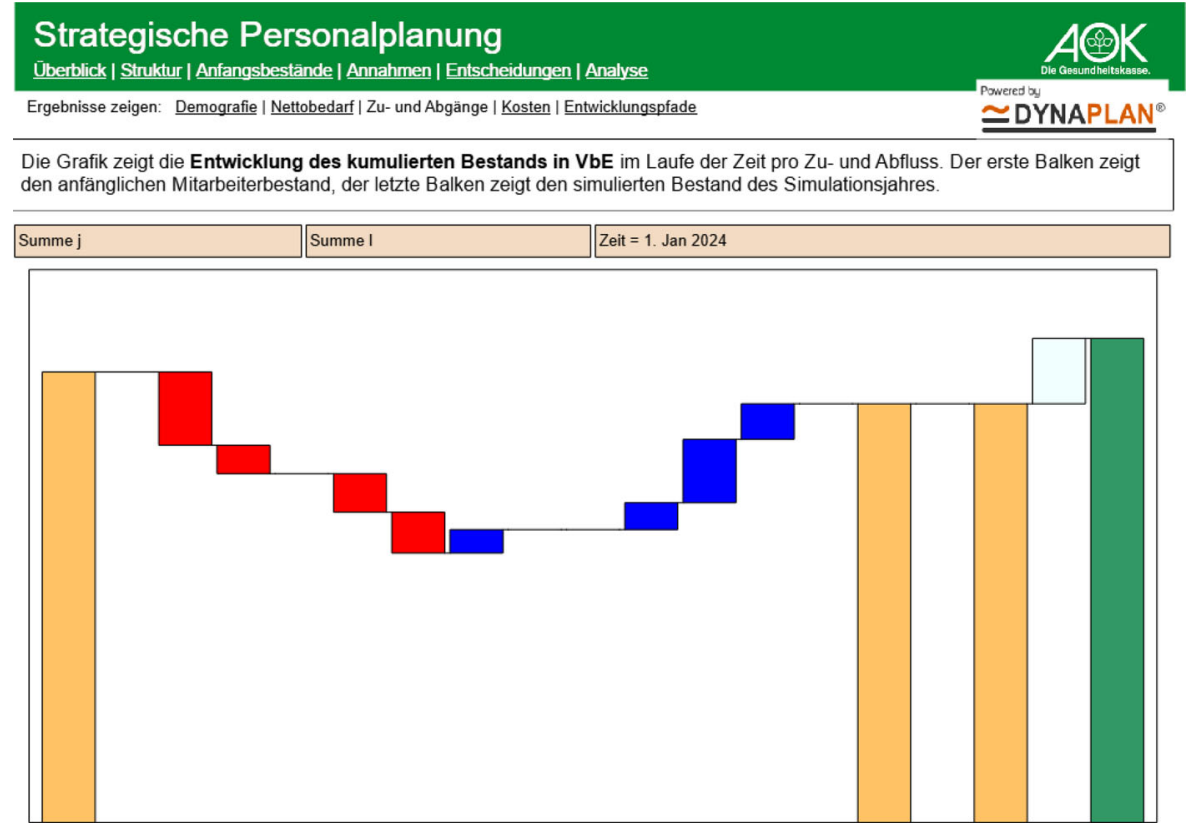

Abb. 5 Das Simulationscockpit mit Dynaplan - Darstellung der Personaldynamik (Zu- und Abgangsbewegungen in einem Wasserfalldiagramm)

\subsection{Durchgängiger Datenfluss auf Basis von SAP und Dynaplan}

Eine konsolidierte Datenbasis ist das zentrale Element für ein gültiges Simulationsergebnis. Wichtig im Umgang mit den Daten ist daher ein durchgängiger transparenter Datenfluss. Die Daten aus dem HR-Basissystem werden dabei in einem ersten Schritt im Fall der AOK Hessen in dem SAP BW mittels Datenextraktion zur Verfügung gestellt. Auf dieser Basis können bereits erste analytische Aufgaben abgebildet werden (z. B. Reporting mit Hilfe von Kennzahlen mit Zeitbezug auf die Vergangenheit im Rahmen einer Zeitreihenanalyse sowie dem aktuellen Zeitpunkt). Im nächsten Schritt werden die Daten aggregiert über eine Query mit Hilfe eines zertifizierten Webservices in das Simulationsmodell übernommen. Die Aggregation erfolgt dabei über zwei Sichtweisen. Auf der einen Seite erfolgt eine Zusammenführung aus der Sicht einer Funktionsbetrachtung (wie z. B. Mitarbeiter in den Funktionen „Leistungsbringer managen“ oder „Kundenbetreuung“). Die zweite Sicht bildet die Zusammenführung nach Qualifikationslevel (wie z. B. „Sachbearbeiter“ oder „Spezialist"). Im Rahmen der strategischen Personalplanung wird das Ergebnis auch als Jobcluster bezeichnet. Für die Bildung haben sich in der Praxis mehrere Gestaltungsprinzipien bewährt (vgl. Berendes et al. 2011).

Der Prozess der strategischen Szenarioplanung endet in der Regel mit einem robusten Planszenario. Die notwendigen Initiativen sind abgeleitet. Für das operative Management erfolgt in Form von Eckwerten eine Vorsteuerung der steuerungsrelevanten Kennzahlen. Für einen besseren Abgleich können diese Werte in das 
Analyticssystem (hier SAP BW) zurückgespielt werden. Damit entsteht eine sinnvolle Ergänzung des bestehenden Reportings um eine vorwärtsgerichtete Sichtweise. Wenn auch nur auf einer aggregierten Ebene (Ebene Jobcluster) können die eingeleiteten Maßnahmen auf der operativen Ebene abgeglichen werden. Bei der AOK wird das im Aufbau befindliche HR-Kennzahlensystem konkret in den folgenden Kennzahlenfeldern ergänzt:

A. Mitarbeiterstruktur und Vielfalt,

1. Altersprofil (Durchschnittsalter; MA über 50 Jahre; Verhältnis <45/>45),

2. Versichertenstruktur im Verhältnis zur Mitarbeiterstruktur,

3. Gender,

4. Entwicklung von Bedarf und Bestand mit Standardmaßnahmen gemessen in Kapazitätseinheiten (Vollbeschäftigungseinheiten),

5. Personalbewegungen,

6. Renteneintritte und Pensionierungen.

B. Ausbildung \& Entwicklung,

1. Anzahl der Auszubildenden und Studienteilnehmer,

2. Externe Einstellungen,

3. Interne Entwicklungen.

C. Kosten,

1. Personalkostenentwicklung.

Nicht alle Kennzahlenfelder können aus der Sicht der vorliegenden strategischen Personalplanung ergänzt werden. Offen bleibt beispielsweise das Feld Mitarbeiterengagement oder Mitarbeiterattraktivität. Hier könnte allenfalls eine gewünschte höhere Mitarbeiterbindung sich in den Annahmen der Simulation über die freiwilligen Abgänge darstellen. Die Aufstellung der Felder ist daher nur ein Auszug aus dem Gesamtsystem.

Die Abb. 6 zeigt einen idealtypischen Datenfluss zwischen den einzelnen HRFunktionen als ein integriertes System. Dabei darf jedoch nicht unerwähnt bleiben, dass gerade aufgrund der Abbildung der Mitarbeiterbedarfe auch Daten aus den

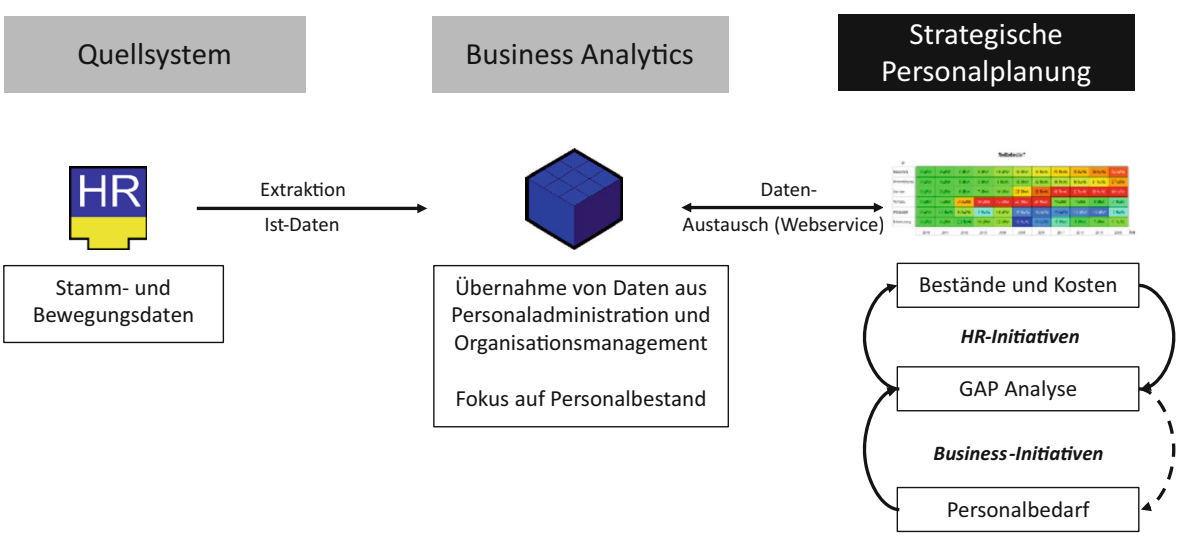

Abb. 6 Durchgängiger Datenfluss 
Geschäftsbereichen und damit außerhalb der Personalsysteme für die Simulation benötigt werden. Die Integration hat damit auch Grenzen.

\section{Nutzen und Ausblick}

Eine Extrapolation von bekannten Mustern als Bestandteil von Analytics-Lösungen kann nur bedingt helfen, die Zukunft zu meistern. Nicht immer ist die Vergangenheit ein guter Indikator für die Zukunft. Die Zukunft ist unbestimmt und kann auf lange Sicht nicht prognostiziert werden. Die hier vorgestellte Kombination aus Analytics und Szenariosimulation bei der AOK erlaubt jedoch eine Vorbereitung auf mögliche Zukunftszustände und ist mit einem Plan zur Stelle, wenn sich entwickelte Umfeldszenarien oder Teile davon am Horizont abzeichnen.

Notwendig ist eine robuste, pragmatisch anzuwendende und in hohem Maß automatisierte Szenariomethodik, die zum Lösen von bewährten Mustern zwingt und die Konzentration auf die wesentlichen Dinge fördert.

Die Integration dieser Methodik in den strategischen Planungsprozess schafft die Grundlage dafür, dass das Denken in Szenarien keine Übung bleibt, sondern zu einem lebenden und gelebten Bestandteil im Rahmen der strategischen Planung wird. Dazu trägt auch der durchgängige Datenfluss bei der AOK zwischen Simulationsmodell und HR Analytics System bei, welcher die Zeit für die Initialisierung der Simulation für einen neuen Planungslauf soweit wie möglich reduziert. Es bleibt jedoch die wichtige und teilweise zeitintensive Diskussion mit den Fachbereichen über Annahmen bestehen.

Aus Sicht der AOK wird mit der strategischen Personalplanung ein Quantensprung im HR-Management erreicht. Es ist erstmals gelungen, ganzheitliche, komplexe Personalszenarien - und nicht nur reduziert auf Teilaspekte des Personalbestands wie Demographie - für alle Anspruchsgruppen als Grundlage für strategische Entscheidungsprozesse transparent zu simulieren. Dies hat den Vorteil, dass das Planungstool auch Akzeptanz in der Unternehmensführung gefunden hat, weil es an die Unternehmensstrategie gekoppelt ist und eine schlüssige Planung zulässt. Die Ergebnisse sind transparent. Diskussionen über Detailfragen werden versachlicht und beschleunigt, da die Wirkungsmechanismen sehr schnell veranschaulicht und für alle Diskussionsteilnehmer nachvollziehbar werden. Vor der Einführung der strategischen Personalplanung hatten die Verantwortlichen teilweise sehr unterschiedliche Vorstellungen über die Auswirkungen der strategischen Ausrichtung auf den Personalbestand. Auf Basis der mit dem Simulationsmodell errechneten Zahlen ergab sich für alle Beteiligten ein gemeinsam getragenes Zukunftsbild. Die Bereiche Personal, Organisation und Prozesse, Technologie, Gender sowie Immobilien haben ausgehend von dem gemeinsamen Zukunftsbild sehr frühzeitig aufeinander abgestimmte Maßnahmen entwickelt und bewerten die Maßnahmen im Hinblick auf die Wirksamkeit mit dem Simulationsmodell.

Die Kommunikation der Erkenntnisse aus den Szenarien erfolgt heute im Rahmen von Managementmeetings in der Regel in Präsentationsform mit anschließender Diskussion. In der Zukunft soll die Auseinandersetzung mit den Szenarien auf der Seite der Führungskräfte noch weiter gestärkt und als Regelprozess verankert wer- 
den. Unter Rückgriff auf die neuen Möglichkeiten in Dynaplan von freigebenden Simulationsmodellen kann der Planer vordefinierte Szenarien und ausgewählte Stellhebel als „Spielmodell“ zur Verfügung stellen. An die Stelle von statischen Folien können Management und HR sich als Business Partner spielerisch mit den Szenarien auseinandersetzen und selber die Dynamik explorieren. Die aktive Simulation soll dabei eine Korrektur von schnellen, intuitiven Vorhersagen ermöglichen (vgl. Kahneman 2011). Zudem entfällt eine mühsame Erstellung umfassender Foliendokumentationen. Der Planer gewinnt über diese Maßnahme wieder Zeit für weitere wertschöpfende Aufgaben und erhält zudem eine neue Qualität an Rückmeldungen zu den Planungsprämissen seitens des Managements. Effizienz und Effektivität werden hiermit permanent optimiert und unterstützen in hohem Maß die Planungsprozesse der AOK Hessen.

Open Access Dieser Artikel wird unter der Creative Commons Namensnennung 4.0 International Lizenz (http://creativecommons.org/licenses/by/4.0/deed.de) veröffentlicht, welche die Nutzung, Vervielfältigung, Bearbeitung, Verbreitung und Wiedergabe in jeglichem Medium und Format erlaubt, sofern Sie den/die ursprünglichen Autor(en) und die Quelle ordnungsgemäß nennen, einen Link zur Creative Commons Lizenz beifügen und angeben, ob Änderungen vorgenommen wurden.

\section{Literatur}

Berendes K et al (2011) Strategische Personalplanung. Zukunft gestalten. Wirtschaftsverlag NW, Bremerhaven

Dörner D (1989) Die Logik des Misslingens. Strategisches Denken in komplexen Situationen, 10. Aufl. Rowohlt, Reinbek

Drucker P (1993) Managing for results. HarperCollins, New York

Kahneman D (2011) Thinking, fast and slow. Macmillan, New York

Milling PM (2002) Understanding and managing innovation processes. Syst Dyn Rev 18(1):73-86

Wegerich C (2015) Strategische Personalentwicklung in der Praxis. Springer, Berlin, Heidelberg 